\title{
Use of non-steroidal anti-inflammatory drugs in the treatment of bacterial respiratory tract infections of lambs
}

\author{
Mehmet Nihat Ural* \\ Department of Experimental Animal Center, Pendik Veterinary Control Institute, Bati Mah, Istanbul, Turkey
}

Received: 21 June 2021

Accepted: 23 July 2021

*Correspondence:

Dr. Mehmet Nihat Ural,

Email: nihatural@yahoo.com

Copyright: $\odot$ the author(s), publisher and licensee Medip Academy. This is an open-access article distributed under the terms of the Creative Commons Attribution Non-Commercial License, which permits unrestricted non-commercial use, distribution, and reproduction in any medium, provided the original work is properly cited.

\begin{abstract}
Respiratory tract infections, which are common in sheep farming, involve interactions between the host's immune system, pathogenic microorganisms such as viruses, bacteria, parasites and environmental conditions. Respiratory diseases can affect sheep of any age and breed. However, acute bacterial and viral pneumonia is most common in those aged three to twelve months due to decreased maternal antibody titers. Although lower respiratory tract infections initially started as a condition caused by a single agent, many cases progress to a complex disease involving both bacterial and viral components, making the diagnosis of the disease complicated by the emergence of secondary infections. Although the inflammatory reaction that occurs in respiratory infections is part of the normal healing process, an extreme reaction can impair healing and severely damage the airways. It is optimal to limit the inflammatory reaction by removing the harmful agent, isolating the damaged tissue, and controlling this tissue. In cases of death due to acute pneumonia, antibiotic drugs are used to prevent further deaths and to treat the herd. It has been found that NSAIDs reduce the release of toxic peroxide which causes the proliferation of fibroblasts and the deposition of collagen in inflammatory processes associated with the respiratory tract. It had been reported that clinical findings improve more rapidly when NSAIDs (non-steroidal anti-inflammatory drugs) were used together with antibiotics in respiratory system infections. In this review, the importance of using NSAIDs in the respiratory tract infections of sheep has been mentioned.
\end{abstract}

Keywords: Bacterial pneumonia, Lamb, NSAIDs, Treatment

\section{INTRODUCTION}

Respiratory tract diseases, which are common in sheep breeding and are caused by the interaction of host, environmental factors and various microorganisms are divided into two categories as upper and lower respiratory tract diseases. ${ }^{1,2}$ While diseases such as nasal myiasis, enzootic nasal tumors, nasal foreign bodies and rhinitis, which are mainly limited to the sinus, nostrils and nasal cavity and have a low incidence, constitute upper respiratory tract diseases, chronic viral diseases such as bacterial, viral, mycotic and parasitic pneumonias, lung abscesses, aspiration pneumonia, maedi-visna constitute lower respiratory tract infections. ${ }^{1,3}$
Pneumonia is inflammation of the lung parenchyma tissue. It is clinically manifested by an increase in respiratory rate, changes in respiratory depth and character, coughing and abnormal breath sounds on auscultation. ${ }^{4}$ The most common types of infection in the lower respiratory tract of lambs are acute and chronic enzootic pneumonia. It has been reported that acute enzootic pneumonia is caused mainly by Mannheimia haemolytica, while chronic enzootic pneumonia is caused by various viral infection factors, especially $M$. Ovipneumoniae and especially Parainfluenza virus type-3 (PI-3). ${ }^{3,5}$

Unlike steroids, which inhibit all arachidonic acid products, NSAIDS have a narrower anti-inflammatory 
spectrum as cyclooxygenase inhibitors. However, NSAIDs have a wider margin of safety that largely compensates for their narrower spectrum. NSAIDs act mainly to inhibit the biosynthesis of prostaglandins. They also inhibit kallikrein activity and kinin formation and also pharmacologically antagonize the tissue effects of kinins, prostaglandins and slow-reacting anaphylaxis substance (SRS-A). ${ }^{6}$ NSAIDs have been shown to have the ability to improve gas exchange in pneumonic calves under experimental conditions. $^{7}$ There are numerous field studies showing the benefits of NSAID therapy in bovine respiratory diseases. ${ }^{8-}$ 11

\section{Bacterial pneumonia}

\section{M. haemolytica}

M. haemolytica, which forms part of the normal flora of the upper respiratory tract in ruminants, is a gram-negative, aerobic, inert and non-spore-forming rod-shaped bacterium. $M$. haemolytica, which is the main bacterial cause of acute pneumonic pasteurellosis and chronic nonprogressive pneumonia, has two biotypes, biotype A and biotype T. Biotype A causes septicemia in young lambs and pneumonia in sheep of all ages, while biotype $\mathrm{T}$ causes infections that usually affect the upper digestive tract and lungs in young adults. ${ }^{12-14} \mathrm{M}$. haemolytica has several virulence factors such as lipopolysaccharide (LPS), leukotoxin (LKT), neuraminidase, capsular polysaccharide and proteases. ${ }^{15}$ Each serotype of $M$. haemolytica produces a characteristic polysaccharide capsule to protect the organism and prevent phagocytosis by macrophages and polymorphonuclear leukocytes. The cell capsule is an important virulence factor that plays a vital role in the pathogenicity of pathogenic bacteria and the establishment of infection. ${ }^{16}$ Although the source of infection is usually the carrier adults, the agent can survive for a long time in the environment, litter and water. M. haemolytica infections are most common during the spring and summer months, particularly between May and July and more particularly during the autumn and winter months. ${ }^{3}$ Clinical manifestations vary. While cough and oculonasal discharge are observed in mild cases, fever of $40.4-42^{\circ} \mathrm{C}$, anorexia, hyperpnea, dyspnea, serous tear-nasal discharge, pathological sounds on auscultation and sudden death may be observed in severe cases. The clinical course of the disease in untreated animals usually results in death within 12 hours, while some animals may survive for up to three days. In chronic cases, the disease may recur after recovery and growth retardation may occur in animals. The disease often causes septicemia with pulmonary involvement in lambs younger than 12 weeks of age. Morbidity in lambs can be up to $40 \%$, while the mortality rate is usually between $5-10 \% .^{3,4,17}$

\section{Pasteurella multocida}

P. multocida, which causes pneumonic and septicemic pasteurellosis in sheep, is a gram-negative bacterium. Pasteurella species are often found as part of the normal flora of the oral cavity, nasopharynx and upper respiratory tract. Pneumonic pasteurellosis is a disease mainly seen in animals with weak pulmonary defense mechanisms. The exposure of the sheep to physical stress or unfavorable environmental conditions plays an important role in the development of the disease. Infections are more serious due to the fact that lambs are more sensitive to the agent. Infected animals show clinical signs such as high fever, shortness of breath, cough, foamy and runny nose..$^{16,18}$

\section{Histophilus somni}

H. somni is a gram-negative bacterium that occurs with various clinical syndromes such as thrombotic meningoencephalitis, pleuritis, polysynovitis, arthritis, bronchopneumonia, septicemia, myocarditis, otitis media and causes a disease complex called histophylosis in cattle and sheep. As a result of DNA hybridization studies, $H$. somnus, H. ovis and Haemophilus agni were accepted as a single species, H. somni, based on the sequencing of $16 \mathrm{~S}$ rRNA and rpoB genes. The sheep strain of $H$. somni can infect cattle and goats, while the cattle strain cannot infect sheep 3. ${ }^{19-21}$

\section{Bordetella parapertussis}

The B. parapertussis strain consists of two genetically different strains, B. parapertussis hu (Bpp hu), which causes infection in humans and B. parapertussis ov (Bpp ov), which is isolated from sheep only. Bpp hu causes pertussis in humans, while Bpp ov has been isolated from both healthy and sheep lungs with chronic nonprogressive pneumonia. $^{22,23}$ B. parapertussis causes mild pyrexia, bronchopneumonia and tracheobronchitis in lambs by damaging alveolar macrophages and epithelial cells of bronchioles and alveoli. Damage to the tracheal epithelium caused by $B$. parapertussis infections may lead to secondary M. haemolytica infections. ${ }^{3,22}$

\section{Streptococcus zooepidemicus}

S. zooepidemicus is an animal pathogen that mainly affects horses, causing diseases in the upper respiratory tract, uterus, navel and wounds. ${ }^{24}$ It can cause mastitis in cows and septicemia in other animals such as rabbits and pigs. ${ }^{25}$ It causes fibrinous pericarditis, fibrinous pleuritis and pneumonia in sheep. Common symptoms of infections caused by $S$. zooepidemicus include pyrexia, mucopurulent nasal discharge and shortness of breath. ${ }^{26}$

\section{Erysipelothrix rhusiopathiae}

E. rhusiopathiae is a gram-positive rod-shaped pathogenic bacterium commonly found in nature and found in domestic animals such as sheep, horses, cattle, chickens, turkeys, dogs, cats and marine animals such as crabs and fish. ${ }^{27}$ E. rhusiopathiae is a facultative intracellular pathogen and replicates in phagocytic cells. The most common clinical finding in sheep is polyarthritis, typically seen in 2-6 month old lambs. In addition, it causes 
cutaneous infection, endocarditis, pneumonia and septicemia. $^{28}$

\section{OTHER POTENTIAL BACTERIAL FACTORS}

Apart from the primary factors mentioned above in sheep, it has been reported to cause respiratory system infections in opportunistic bacteria such as Escherichia coli, Streptococcus spp., Klebsiella spp., Pseudomonas spp. and Neisseria spp./Neisseria cuniculi. ${ }^{3}$

\section{TREATMENT}

The basic principle in treatment is to reduce the amount and viscosity of secretion and facilitate its excretion. In addition, adequate oxygenation of the blood and carbon dioxide excretion should be ensured, pulmonary inflammation should be alleviated, infectious agents should be treated effectively, bronchoconstriction should be eliminated and supportive care should be provided for the transport of respiratory gases. In the treatment of respiratory system diseases, antitussives, antimicrobials, bronchodilators, diuretics, expectorants and other drugs are used. $^{4,29}$

The basic therapeutic strategy in the treatment of bovine respiratory tract infections consists of a treatment protocol in which an antibiotic effective against pathogens and a NSAIDs are used together against the harmful effects of inflammation. ${ }^{30}$ However, although there is no licensed NSAID for the treatment of respiratory system diseases in sheep in the world, the data obtained from the results of studies on infections of organs or systems other than respiratory system in small ruminants. There was evidence that the use of NSAIDs may be beneficial in respiratory tract infections. ${ }^{31}$ It had been determined that the use of NSAIDs together with antibiotics in infected animals eliminated free oxygen radicals, inhibited phosphorylation, blocked the release of kinins and proteoglycan degradation, and also prevented the migration and adhesion of neutrophils. $^{32}$

It had been shown that the application of antioxidants such as vitamins $\mathrm{E}$ and $\mathrm{C}$ together with antibiotics and NSAIDs in the treatment of respiratory tract infections of cattle effectively limits the development of oxidative stress and inflammatory process and causes an increase in resistance to disease. ${ }^{33}$ It had been found that vitamin $\mathrm{E}$ administration can inhibit the inflammatory process by inhibiting the production of proinflammatory cytokines, reducing the production of acute phase proteins and attenuating the inflammatory response of epithelial cells in the lungs. It was thought that the findings obtained from the application of antioxidants such as vitamins $\mathrm{E}$ and $\mathrm{C}$ may be a guide for the development of an effective adjuvant therapy. ${ }^{34}$ Apart from vitamins $\mathrm{E}$ and $\mathrm{C}$, it has been shown that vitamin $\mathrm{D}$ can also be used as adjuvant therapy in the treatment of infection. ${ }^{35,36}$ It has been stated that cathelicidin synthesis was decreased in the bronchial epithelium in respiratory tract infections and vitamin D can be used to correct this situation. ${ }^{37}$ Due to the reduced feed consumption due to the disease, it was recommended to increase the protein and energy ratio in the diet of sick animals, as well as supplementation of vitamin $\mathrm{A}, \mathrm{Zn}, \mathrm{Cu}$, $\mathrm{Fe}$ and I, apart from the above-mentioned vitamins. ${ }^{38}$

Mucolytics reduce the viscosity of sputum, making it easier to clean sputum. It has been stated that bromhexine can increase the concentration of antibiotic drugs in the lung tissue by changing the permeability of the mucosa as well as improving gas exchange by reducing respiratory tract obstruction and persistent mucus accumulation. Bronchodilators may be valuable in reducing the severity of clinical manifestations by preventing reduced tidal volume caused by pulmonary edema and inflammation, and by increasing gas exchange in animals with pulmonary lesions. ${ }^{39}$ Methylxanthines, such as theophylline and aminophylline are effective bronchodilators in species other than cattle (and possibly dogs); however, these drugs have a relatively narrow therapeutic index and are less effective than b2-agonists. ${ }^{29}$ Clenbuterol, one of the $\beta 2$ agonists, can be used in horses and cattle at a dose of 0.8 $\mu \mathrm{g} / \mathrm{kg}(\mathrm{PO} / \mathrm{IV} / \mathrm{IM}, \mathrm{BID}){ }^{40}$

In the experimental infection study conducted by Gilmour et al (1982) one group of specific pathogen-free lambs was administered long-acting oxytetracycline at a dose of 20 $\mathrm{mg} / \mathrm{kg} 24$ hours before exposure to M. haemolytica via the aerosol route and the other group was treated with 20 $\mathrm{mg} / \mathrm{kg}$ of long-acting oxytetracycline and compared with similarly infected but untreated lambs. It was determined that the symptoms of pneumonia and lamb deaths were delayed in the lambs treated with the drug before the exposure and the lung lesions detected in the lambs in the treated group after the infection were significantly less than in the untreated lambs. As a result of the study, it had been shown that oxytetracycline application is effective in $M$. haemolytica infections and better results are obtained when applied after infection. ${ }^{41}$

In a field study conducted in England by Sarginson et al (1995), an epidemic that caused the death of 8 lambs within a period of 1 week in a herd of 188 lambs at 5 weeks of age and was thought to be septicemic pasteurellosis, half of the lambs were administered subcutaneously with a single dose of $10 \mathrm{mg} / \mathrm{kg}$ tilmicosin and the other half was administered intramuscularly as a single dose of $20 \mathrm{mg} / \mathrm{kg}$ dose of oxytetracycline was administered. It was determined that clinical improvement occurred within 2 days in both lamb groups and there was a rapid increase in daily live weight gains. ${ }^{42}$ The most commonly used antibiotic for the treatment of pasteurellosis is oxytetracycline administered by slow intravenous injection at a dose of $10 \mathrm{mg} / \mathrm{kg}$. Following this initial administration, intramuscular administration at a dose of $10 \mathrm{mg} / \mathrm{kg}$ for 3 to 4 consecutive days or a single dose of $20 \mathrm{mg} / \mathrm{kg}$ long-acting oxytetracycline has been reported to be effective. Tilmicosin (5-10 mg/kg, SC, single dose), florfenicol (20 $\mathrm{mg} / \mathrm{kg}$, IM, 2 times with 48 hours intervals), danofloxacin $(6 \mathrm{mg} / \mathrm{kg}, \quad \mathrm{SC})$. It has been shown that licensed 
antimicrobial drugs such as single dose) can also be effective in sheep. ${ }^{31,43,44}$

\section{PROTECTION}

Pneumonia in sheep of all ages was largely caused by the combination of infectious agents, unsuitable physical environment and physiological stress. For the prevention and control of respiratory system diseases of sheep, the barns should be adequately ventilated and wet and windy places that cause stress should be avoided. Young and adult animals should not be kept in the same area, animals should be grouped according to age, size and origin. The animals that will join the herd must be quarantined.,31

There are different reports on the protection of vaccination against factors causing respiratory system diseases in sheep. It has been reported that the PI-3 vaccine does not provide adequate protection and does not lead to a significant decrease in the incidence of pneumonia. It was stated that $M$. haemolytica may not provide sufficient protection against infections caused by serotypes that are not included in the vaccine due to the presence of a wide variety of serotypes. Vaccination of ewes against pneumonia caused by $M$. haemolytica four to six weeks before lambing ensured that lambs acquire antibody protection from colostrum for the first three to four weeks of life. Vaccination was not recommended before the age of three weeks as colostral antibodies affect active immune development. It was recommended to administer the second dose 4 to 6 weeks after the first vaccination at the age of three weeks, when the risk of disease increased and to repeat the vaccination every six or twelve months, depending on the presence of risk factors for the disease. . $^{3,31,45-47}$

Although its effectiveness against respiratory system infections in sheep has not been proven, long-acting metaphylactic antibiotics such as oxytetracycline and tilmicosin can be applied. ${ }^{31}$

\section{NSAIDs}

NSAIDs are drugs with analgesic, antipyretic and antiinflammatory effects. The term non-steroid is used to distinguish these drugs from steroids with similar effects. NSAIDs targeting central and peripheral pain mediators are widely used in veterinary medicine as well as human medicine. With the introduction of new NSAIDs since the 1990 s, their use in veterinary practice has increased significantly. ${ }^{48,49}$ NSAIDs act by inhibiting the action of cyclooxygenase (COX) enzyme responsible for the synthesis of prostaglandins, which play a role in inflammation, fever and pain in the body. ${ }^{50,51}$

\section{COX enzyme}

It has been shown that there are two different isoforms: constitutive (COX-1) and inducible (COX-2). COX-1 is found in most cells and tissues including vascular endothelium, monocytes, gastrointestinal epithelial cells and platelets. COX-1 isoenzyme plays an important role in many physiological events such as protection of gastric mucosa and regulation of renal blood flow through prostaglandin E2 and prostaglandin I2 and platelet aggregation through thromboxane A2. In contrast, COX-2 is found constitutively in only a few tissues such as nerve, reproductive and kidney. It is an enzyme that is induced in tissues in inflammatory reactions. It takes part in the production of prostaglandins that cause inflammation, fever and pain. ${ }^{49,52-54}$

Inflammatory stimuli cause arachidonic acid formation from phospholipids in the cell membrane via the phospholipase A2 enzyme. Arachidonic acid, a 20-carbon unsaturated fatty acid is the source of all eicosanoids (prostanoids). Arachidonic acid is first converted to prostaglandin (PG) $\mathrm{G} 2$ and then to $\mathrm{PGH} 2$ by $\mathrm{COX}$ isoenzymes, then PGD2, PGE2, PGF2 $\alpha$, PGI2 (prostacyclin) and thromboxane A2 (TXA2) are produced by a series of sequential reactions. NSAIDs inhibit the COX isoenzyme, reducing the synthesis of these COX products that mediate inflammation. Glucocorticoids with stronger anti-inflammatory effects, on the other hand, have an inhibitory effect on the phospholipase A2 enzyme, reducing the synthesis of not only cyclooxygenase products, but also lipoxygenase products. ${ }^{51-53,55}$

Due to their weak acidic chemical structure, NSAIDs are well absorbed following oral administration. They are highly bound to plasma proteins and the amount of free drug is relatively low. Because it is the free fraction of the drug that is of therapeutic importance, careful adjustment of dosage is required in animals with abnormal serum albumin concentrations. Toxicity may occur when hypoalbuminemic animals are given the usual dose, but this is often not a problem as the free fraction of the drug is rapidly metabolized or excreted. Most of the drugs in this group are metabolized in the liver and excreted by the kidneys through the urine. Significant dose reductions may be required in both pediatric and geriatric animals due to age-related differences in distribution volumes and metabolisms..$^{50,56}$

\section{Use of NSAIDs in respiratory tract infections}

In respiratory infections, leukocytes collect in the lungs, helping to remove invading organisms through phagocytic clearance. However, in some cases, the normal inflammatory response can be destructive as well as defensive. Acute respiratory infections, regardless of location are histologically characterized by protein exudation, proteinaceous eruptions and a large number of neutrophils. Although neutrophil influx is part of the host's natural defense against an invading organism, it has recently led to the view that neutrophils can have devastating effects on pulmonary cells and interstitial tissues. The source of this destructive potential is neutrophil elastase, a proteolytic enzyme synthesized in azurophil granules. It has been determined that this enzyme 
scrapes the bronchial epithelium, reduces ciliary pulsation, stimulates excessive mucus secretion, causes mucus retention, bacterial proliferation and recurrent infections. Neutrophil elastase also stimulates epithelial cell interleukin- 8 secretion and produces other chemoattractant cleavage products leading to greater neutrophil uptake. It also impairs host defense by damaging the main opsonophagocytic receptor on neutrophils and weakening the efficacy of immunoglobulins. Although the inflammatory reaction that occurs in respiratory infections is part of the normal healing process, excessive reaction can impair healing and seriously damage the respiratory tract. It is most appropriate to limit the inflammatory reaction by eliminating the harmful agent, isolating the damaged tissue and controlling this tissue. ${ }^{57-59}$

During inflammatory reactions, the activity of the COX-1 enzyme, which is a structural enzyme, increases 2-3 times, while the activity of the inducible form of COX-2 enzyme can increase more than 20 times. Endotoxins are the most potent inducers of COX-2 synthesis in monocytes and alveolar macrophages. Also, proinflammatory cytokines such as IFN and tumor necrosis factor (TNF) can induce COX-2 in macrophages and IL-1 in endothelial cells. ${ }^{60}$ TNF stimulates the production of PGE2 with its collagenase activity and is responsible for the destruction and restructuring of connective tissue in inflammatory reactions. In addition, it has been determined that TNF exerts a proinflammatory effect through the induction of COX-2 activity and prostanoid synthesis. ${ }^{61-63}$ NSAIDs have been found to reduce the release of toxic peroxide in respiratory-related inflammatory processes, causing the proliferation of fibroblasts and the accumulation of collagen, as well as to inhibit the increase of nitric oxide synthase production in lung macrophages, weakening the destructive effect of these cells on the bronchial mucosa. The antitoxic activity of NSAIDs is based on inhibition of malondialdehyde (MDA) production. ${ }^{32}$

It has been shown that NSAIDs can affect cytokine production and exert some anti-inflammatory effects by suppressing monocyte/macrophage-derived inflammatory mediators. For example, aspirin has been found to inhibit TNF production in mouse macrophages. ${ }^{64}$ In the study conducted by Bednarek et al (2003) on calves with enzootic bronchopneumonia, three equal groups were formed and oxytetracycline and meloxicam were administered to the first group, oxytetracycline and flumethasone to the second group, and only oxytetracycline to the third group (control). It was found that the animals in the first group performed significantly faster with improvement and a faster normalization in the clinical disease index score (cough, runny nose, dyspnea, depression and anorexia) compared to the other groups. ${ }^{9}$

\section{CONCLUSION}

In the etiology of respiratory tract infections that are common in sheep, besides multiple infectious factors such as bacteria, viruses, mycoplasmas and parasites, environmental factors such as weakening of the host's defense system, improper ventilation conditions, stress, poor care and feeding conditions. Respiratory diseases cause serious financial losses for sheep producers due to low live weight gain and death. Disease is also an important issue for animal welfare. The severity of respiratory tract infections correlated with high eicosanoid concentrations. In the studies, it has been reported that clinical findings improve faster when an NSAIDs drug is administered together with antibiotic treatment in respiratory tract infections. There are not a NSAID licensed for use in sheep in the EU, although there are NSAIDs approved for use in painful and inflammatory conditions in sheep in Canada, New Zealand and Australia. In this case, it is possible to use proprietary products for other food-producing species (cattle) by paying attention to the withdrawal period to prevent residue formation.

\section{Funding: No funding sources \\ Conflict of interest: None declared \\ Ethical approval: Not required}

\section{REFERENCES}

1. Chakraborty S, Kumar A, Tiwari R, Rahal A, Malik $\mathrm{Y}$, Dhama K, et al. Advances in diagnosis of respiratory diseases of small ruminants. Veterin Med Int. 2014:508304.

2. Lacasta D, Ferrer L, Ramos J, González J, Heras MDL. Influence of climatic factors on the development of pneumonia in lambs. Small Ruminant Res. 2008;80(1-3):28-32.

3. Bell S. Respiratory disease in sheep. In Pract. 2008;30(4):200.

4. Radostits OM, Gay CC, Hinchcliff KW, Constable PD. Veterinary Medicine E-Book: A textbook of the diseases of cattle, horses, sheep, pigs and goats. 10th ed. Elsevier Health Sciences; 2006.

5. Ettorre C, Sacchini F, Scacchia M, Salda LD. Pneumonia of lambs in the Abruzzo region of Italy: anatomopathological and histopathological studies and localisation of Mycoplasma ovipneumoniae. Vet Ital. 2007;43(1):149-55.

6. Bednarek D, Szymańska-Czerwińska M, Dudek K. Bovine respiratory syndrome (BRD) etiopathogenesis, diagnosis and control. Bird Eye View Veterin Med. 2012:363-78.

7. Weerdt MVD, Coghe J, Uystepruyst C, Deby-Dupont G, Lekeux P. Ketoprofen and phenylbutazone attenuation of PAF-induced lung inflammation in calves. Veterin J. 1999;157(1):39-49.

8. Bednarek D, Kondracki M, Friton G, Trela T, Niemczuk K. Effect of steroidal and non-steroidal anti-inflammatory drugs on inflammatory markers in calves with experimentally-induced bronchopneumonia. Berliner Munchener tierarztliche Wochenschrift. 2005;118(7-8):305-8.

9. Bednarek D, Zdzisinska B, Kondracki M, KandeferSzerszen M. Effect of steroidal and non-steroidal antiinflammatory drugs in combination with long-acting 
oxytetracycline on non-specific immunity of calves suffering from enzootic bronchopneumonia. Vet Microbiol. 2003;96(1):53-67.

10. Lockwood P, Johnson J, Katz T. Clinical efficacy of flunixin, carprofen and ketoprofen as adjuncts to the antibacterial treatment of bovine respiratory disease. Vet Rec. 2003;152(13):392-4.

11. Weingarten A. Mechanisms of action and the role of anti-pyretic and antiinflammatory intervention in the treatment of bovine respiratory disease. Getting it right the first time: best practices in BRD treatment. EBF Marseille. 2009:6-15.

12. Alley MR. Pneumonia in sheep in New Zealand: an overview. N Z Vet J. 2002;50 (3):99-101.

13. Gilmour NJ. Pasteurella haemolytica infections in sheep. Vet Quarterly. 1980;2(4):191-8.

14. Tabatabaei M, Abdollahi AF. Isolation and identification of Mannheimia haemolytica by culture and polymerase chain reaction from sheep's pulmonary samples in Shiraz, Iran. Vet World. 2018;11(5):636-41.

15. Iovane G, Galdiero M, Vitiello M, DeMartino L. Effect of Pasteurella haemolytica outer membrane proteins on bovine neutrophils. FEMS Immunol Med Microbiol. 1998;20(1):29-36.

16. Mohamed R, Abdelsalam E. A review on pneumonic pasteurellosis (respiratory mannheimiosis) with emphasis on pathogenesis, virulence mechanisms and predisposing factors. Bulgar $\mathrm{J}$ Veterin Med. 2008;11(3):139-60.

17. Brogden KA, Lehmkuhl HD, Cutlip RC. Pasteurella haemolytica complicated respiratory infections in sheep and goats. Veterin Res. 1998;29(3-4):233-54.

18. Fernandez S, Galapero J, Gomez L, Perez C, Ramos A, Cid D, et al. Identification, capsular typing and virulence factors of Pasteurella multocida isolates from Merino lambs in Extremadura (Southwestern Spain). Veterinární Medicína. 2018;63(3):117-24.

19. Angen $O$, Ahrens $P$, Kuhnert $P$, Christensen $H$, Mutters R. Proposal of Histophilus somni gen. nov., sp. nov. for the three species incertae sedis 'Haemophilus somnus', 'Haemophilus agni' and 'Histophilus ovis'. Int J Syst Evol Microbiol. 2003;53(5):1449-56.

20. Headley SA, Pereira AHT, Balbo LC, DiSantia GW, Bracarense A, Filho L, et al. Histophilus somniassociated syndromes in sheep from Southern Brazil. Brazil J Microbiol. 2018;49 (3):591-600.

21. Walker R, Biberstein E, Pritchett R, Kirkham C. Deoxyribonucleic acid relatedness among "Haemophilus somnus," "Haemophilus agni," "Histophilus ovis," "Actinobacillus seminis," and Haemophilus influenzae. Int J Systemat Evolution Microbiol. 1985;35(1):46-9.

22. Chen W, Alley M, Manktelow B. Pneumonia in lambs inoculated with Bordetella parapertussis: clinical and pathological studies. N Z Vet J. 1988;36(3):138-42.

23. Porter JF, Connor K, Donachie W. Isolation and characterization of Bordetella parapertussis-like bacteria from ovine lungs. Microbiology (Reading). 1994;140(2):255-61.

24. Lindmark H, Nilsson M, Guss B. Comparison of the fibronectin-binding protein FNE from Streptococcus equi subspecies equi with FNZ from $S$. equi subspecies zooepidemicus reveals a major and conserved difference. Infect Immun. 2001;69(5):3159-63.

25. Efstratiou A, Colman G, Hahn G, Timoney J, Boeufgras J, Monget D. Biochemical differences among human and animal streptococci of Lancefield group $\mathrm{C}$ or group $\mathrm{G}$. J Med Microbiol. 1994;41(2):145-8.

26. Stevenson R. Streptococcus zooepidemicus infection in sheep. Can J Comp Med. 1974;38(3):243-50.

27. Wang Q, Fidalgo S, Chang B, Mee B, Riley T. The detection and recovery of Erysipelothrix spp. in meat and abattoir samples in Western Australia. J Appl Microbiol. 2002;92(5):844-50.

28. Ersdal C, Jørgensen H, Lie KI. Acute and chronic Erysipelothrix rhusiopathiae infection in lambs. Vet Pathol. 2015;52(4):635-43.

29. Kuehn NF. Principles of Therapy of Respiratory Disease. Available at: https://www.msdvetmanual.com/respiratorysystem/respiratory-system-introduction/principles-oftherapy-of-respiratory-disease. Accessed on 5 June 2021.

30. Lekeux P. A therapeutic strategy for treatment of the bovine respiratory disease complex: The rationale for the combination of a nonsteroidal anti-inflammatory drug with an antibiotic. Cattle Pract. 2007;15(2):1159.

31. Scott PR. Treatment and control of respiratory disease in sheep. Vet Clinic North Am. 2011;27(1):175-86.

32. Wernicki A, Stachura R, Hola P, Puchalski A, Dec M, Stegierska D, et al. Efficacy of florfenicol and flunixin followed with vitamin $\mathrm{E}$ and/or $\mathrm{C}$ on selected oxidative and inflammatory mechanisms in young cattle under transport and adaptation stress. Medycyna Weterynaryjna. 2018;74(4).

33. Urban-Chmiel R, Stachura R, Hola P, Puchalski A, Dec M, Wernicki A. Effects of flunixin and florfenicol combined with vitamins $\mathrm{E}$ and/or $\mathrm{C}$ on selected immune mechanisms in cattle under conditions of adaptive stress. Bull Vet Instit Pulawy. 2015;59(2):295-301.

34. Ekstrand-Hammarström B, Österlun C, Lilliehöök B, Bucht A. Vitamin E down-modulates mitogenactivated protein kinases, nuclear factor- $\kappa \mathrm{B}$ and inflammatory responses in lung epithelial cells. Clinic Experiment Immunol. 2007;147(2):359-69.

35. Cheallaigh CN, Keane J, Lavelle EC, Hope JC, Harris J. Autophagy in the immune response to tuberculosis: clinical perspectives. Clinic Experiment Immunol. 2011;164(3):291-300.

36. Rehman P. Sub-clinical rickets and recurrent infection. J Tropic Pediatr. 1994;40(1):58.

37. Yim S, Dhawan P, Ragunath C, Christakos S, Diamond G. Induction of cathelicidin in normal and $\mathrm{CF}$ bronchial epithelial cells by $1,25-$ 
dihydroxyvitamin D3. J Cystic Fibrosis 2007;6(6):403-10.

38. Edwards T. Control methods for bovine respiratory disease for feedlot cattle. Vet Clinic Food Animal Pract. 2010;26(2):273-84.

39. Cusack P, McMeniman N, Lean I. The medicine and epidemiology of bovine respiratory disease in feedlots. Austral Veterin J. 2003;81(8):480-7.

40. EMEA. The European agency for the evaluation of medicinal products veterinary medicines evaluation unit. committee for veterinary medicinal products. Clenbuterol Summ Rep. 2000;2:1-6.

41. Gilmour N, Sharp J, Gilmour J. Effect of oxytetracycline therapy on experimentally induced pneumonic pasteurellosis in lambs. Vet Rec. 1982111(5):97-9.

42. Sargison N, Scott P. Evaluation of antibiotic treatment of respiratory disease, including suspected septicemic pasteurellosis in five-week-old lambs. Agri Pract (USA). 1995.

43. Godinho K, Benchaoui H, Tilt N, Ramage C, Quirie $\mathrm{M}$, Donachie W, et al. Efficacy of danofloxacin in the treatment of pneumonic pasteurellosis in specific pathogen-free lambs. British Medical Journal Publishing Group; 2007.

44. Naccari F, Pellegrino M, Calò M, Licata P, Giofré F, Carli S. Effectiveness and kinetic behaviour of tilmicosin in the treatment of respiratory infections in sheep. Vet Rec. 2001;148(25):773-6.

45. Gonzalez J, Lacasta D, Ferrer L, Figueras L, Abadie G, Heras MDL. Mannheimia haemolytica and Bibersteinia trehalosi serotypes isolated from lambs with ovine respiratory complex in Spain. J Hellenic Vet Med Soc. 2013;64(3):177-82.

46. Lacasta D, Ferrer L, Ramos J, González J, Ortín A, Fthenakis G. Vaccination schedules in small ruminant farms. Vet Microbiol. 2015;181(1-2):34-46.

47. Lehmkuhl H, Cutlip R. Protection from parainfluenza3 virus and persistence of infectious bovine rhinotracheitis virus in sheep vaccinated with a modified live IBR-PI-3 vaccine. Canadian J Comp Med. 1985;49(1):58.

48. Buer JK. Origins and impact of the term 'NSAID'. Inflammopharmacol. 2014;22(5):263-7.

49. Lascelles BDX, McFarland JM, Swann H. Guidelines for safe and effective use of NSAIDs in dogs. Vet Therapeut. 2005;6(3):237.

50. Curry SL, Cogar SM, Cook JL. Nonsteroidal antiinflammatory drugs: a review. J Am Animal Hospit Assoc. 2005;41(5):298-309.
51. Ricciotti E, FitzGerald GA. Prostaglandins and inflammation. Arterioscleros Thrombos Vascul Biol. 2011;31(5):986-1000.

52. Brune K, Patrignani P. New insights into the use of currently available non-steroidal anti-inflammatory drugs. J Pain Res. 2015;8:105.

53. Rao P, Knaus EE. Evolution of nonsteroidal antiinflammatory drugs (NSAIDs): cyclooxygenase (COX) inhibition and beyond. J Pharm Pharmaceut Sci. 2008;11(2):81-110.

54. Simmons DL, Botting RM, Hla T. Cyclooxygenase isozymes: the biology of prostaglandin synthesis and inhibition. Pharmacolog Rev. 2004;56(3):387-437.

55. Kim SJ, Flach AJ, Jampol LM. Nonsteroidal antiinflammatory drugs in ophthalmology. Survey Ophthalmol. 2010;55(2):108-33.

56. Brater DC. Clinical pharmacology of NSAIDs. J Clinic Pharmacol. 1988;28(6):518-23.

57. Stockley RA. Role of inflammation in respiratory tract infections. Am J Med. 1995;99(6):8-13.

58. Cone JB. Inflammation. Am J Surg. 2001;182(6):55862.

59. Lekeux P. BRDC and the modulation of lung inflammation. Veterinary J. 2006.

60. Endo T, Ogushi F, Kawano T, Sone S. Comparison of the regulations by Th2-type cytokines of the arachidonic-acid metabolic pathway in human alveolar macrophages and monocytes. Am J Respirat Cell Molecul Biol. 1998;19(2):300-7.

61. Frölich J. A classification of NSAIDs according to the relative inhibition of cyclooxygenase isoenzymes. Trend Pharmacol Sci. 1997;18(1):30-4.

62. Takashiba S, Dyke TEV, Shapira L, Amar S. Lipopolysaccharide-inducible and salicylate-sensitive nuclear factor(s) on human tumor necrosis factor alpha promoter. Infect Immun. 1995;63(4):1529-34.

63. Vassalli P. The pathophysiology of tumor necrosis factors. Ann Rev Immunol. 1992;10(1):411-52.

64. Shackelford RE, Alford B, Xue Y, Thai SF, Adams DO, Pizzo S. Aspirin inhibits tumor necrosis factoralpha gene expression in murine tissue macrophages. Mol Pharmacol. 1997;52(3):421-9.

Cite this article as: Ural MN. Use of non-steroidal anti-inflammatory drugs in the treatment of bacterial respiratory tract infections of lambs. Int J Basic Clin Pharmacol 2021;10:1149-55. 